\title{
Resistance of erythrocytes to lipid peroxidation in alcoholic patients
}

\author{
N A Punchard, H Senturk, J P Teare, R P H Thompson
}

\begin{abstract}
The ability of erythrocytes to resist lipid peroxidation may be a useful marker of antioxidant status in alcoholic patients, in whom depletion of dietary antioxidants may combine with increased production of free radicals to produce liver damage. There are conflicting reports, however, on the resistance of erythrocytes from alcoholic patients to lipid peroxidation. This study examined the relation between the degree of alcohol induced liver disease and the resistance of erythrocytes to chemically induced lipid peroxidation, measuring lipid peroxidation as malondialdehyde production. Erythrocytes from alcoholic patients with Child's $\mathbf{C}$ cirrhosis had significantly increased resistance to lipid peroxidation compared with both controls $(p<0.001)$ and alcoholic patients with moderate liver disease $(p<0.001)$. There was no difference between alcoholic patients with moderate liver disease and controls. Increased resistance to free radical initiated lipid peroxidation in alcoholic patients is related to liver damage rather than to alcohol abuse alone. This could arise from changes in the lipid composition of the erythrocyte membranes resulting from abnormal liver function. Tests of antioxidant status based upon the resistance of erythrocytes to free radical stress in vitro may therefore be flawed when such changes in membrane lipid composition can occur.
\end{abstract}

(Gut 1994; 35: 1753-1756)

The generation of toxic free radicals from the metabolism of ethanol by hepatic cytochrome $P_{450} \mathrm{II} E 1$ and increased lipid peroxidation have been implicated in alcoholic liver disease. ${ }^{1}$ In addition, the inadequate nutritional intake of alcoholic patients ${ }^{2}$ may lower the concentrations of the nutritionally derived components of the antioxidant defences, such as vitamins $E^{34}$ and $C,{ }^{5}$ zinc, ${ }^{6}$ and selenium, ${ }^{4}$ thereby increasing the vulnerability of the liver to injury.

A simpler and perhaps more relevant alternative to the measurement of individual antixoidants is to measure the sum of their activity by assessing the functional capability of the cellular antioxidant defences. The resistance of erythrocytes to chemically induced lipid peroxidation has been suggested to reflect the resistance of other tissues, ${ }^{78}$ to be a measure of whole body vitamin $E$ status, 910 and to be of use in determining antioxidant deficiencies in alcoholic liver disease. ${ }^{11}$ Certainly, erythrocytes from alcoholic patients may have a lower resistance to lipid peroxidation, measured in vitro either as malondialdehyde production ${ }^{11-13}$ or cell lysis, ${ }^{14}$ which may be caused by lower antioxidant defences 41516 and be related to lipid peroxidation in the liver. ${ }^{13} \mathrm{~A}$ reduced resistance in vivo could account for the increased haemolysis ${ }^{14}$ and abnormal structure $^{17}$ and function ${ }^{18}$ of erythrocytes from alcoholic patients.

Others have shown, however, that erythrocytes from alcoholic patients with liver disease have an increased resistance to chemically induced lipid peroxidation when measured as pentane production. ${ }^{19}$ It is unclear whether this is simply because of the choice of measure of lipid peroxidation or whether the changes in erythrocytes are influenced by the degree of liver damage. The aim of this study was therefore to discover if the resistance of erythrocytes from alcoholic patients to a chemically induced lipid peroxidation is increased when lipid peroxidation is measured by a different technique, namely as malondialdehyde, and to what degree the resistance is related to the severity of liver disease, rather than to alcohol abuse alone.

\section{Methods}

\section{SUBJECTS}

Twenty patients with alcoholic liver disease in St Thomas' Hospital who were not receiving any treatment were investigated. None had consumed alcohol within the last 24 hours. Patients were divided into those with moderate liver damage $(n=10)$, either fatty liver $(n=4$; serum albumin $=44 \cdot 7$ (3.1) $\mathrm{g} / \mathrm{l}$; mean (SD), hepatitis $(n=1$; serum albumin $43 \mathrm{~g} / \mathrm{l})$ or Child-Pugh Class A or B cirrhosis $(n=5$; serum albumin $33.8(8 \cdot 1) \mathrm{g} / \mathrm{l})$, and severe (C cirrhosis $)$ disease $(n=10$; serum albumin $25.6(2 \cdot 7) \mathrm{g} / \mathrm{l})$. Cirrhosis was confirmed histologically.

None had markers of hepatitis B infection. Tests for hepatitis $C$ were not performed. The control group were 10 healthy laboratory subjects with no history of liver diseases or alcohol abuse, currently consuming less than $10 \mathrm{~g}$ alcohol per week, and who had not taken any drugs or consumed any alcohol within the previous 24 hours. The ratio of men to women was $7: 3$ in each group. The age range for the alcoholic group was 26-65 and that for controls 22-62. Approval for this study was obtained from the ethical committee of St Thomas' Hospital, and all patients gave informed consent. 


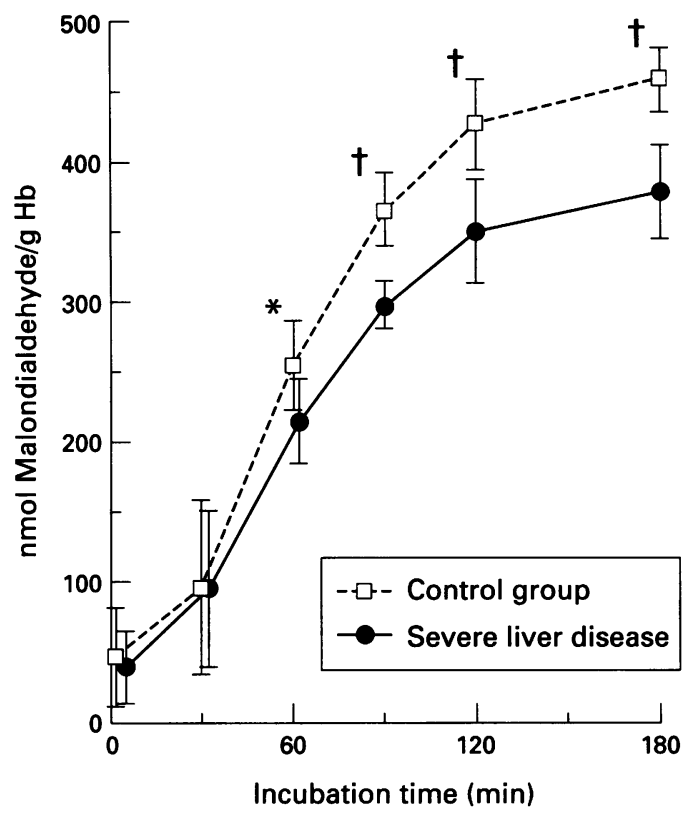

Figure 1: Time course of malondialdehyde production in $t-B H P$ stressed erythrocytes from controls and alcoholic patients with severe liver disease. Data shown as mean (SD). ${ }^{\star}=p<0.02 ; \dagger=p<0.001$.

PREPARATION OF ERYTHROCYTES

Ten $\mathrm{ml}$ heparinised venous blood was centrifuged at $4^{\circ} \mathrm{C}$ at $1400 \mathrm{~g}$ for 15 minutes, the plasma and buffy coat discarded, and the erythrocytes washed three times by resuspending them in phosphate buffered saline (15 mmol $1^{-1}$ phosphate buffer, $139 \mathrm{mmol}^{-1}$ sodium chloride, $\mathrm{pH} 7.4$ containing $1 \mathrm{mmol}$ $1^{-1}$ sodium azide) (PBS), centrifuging at

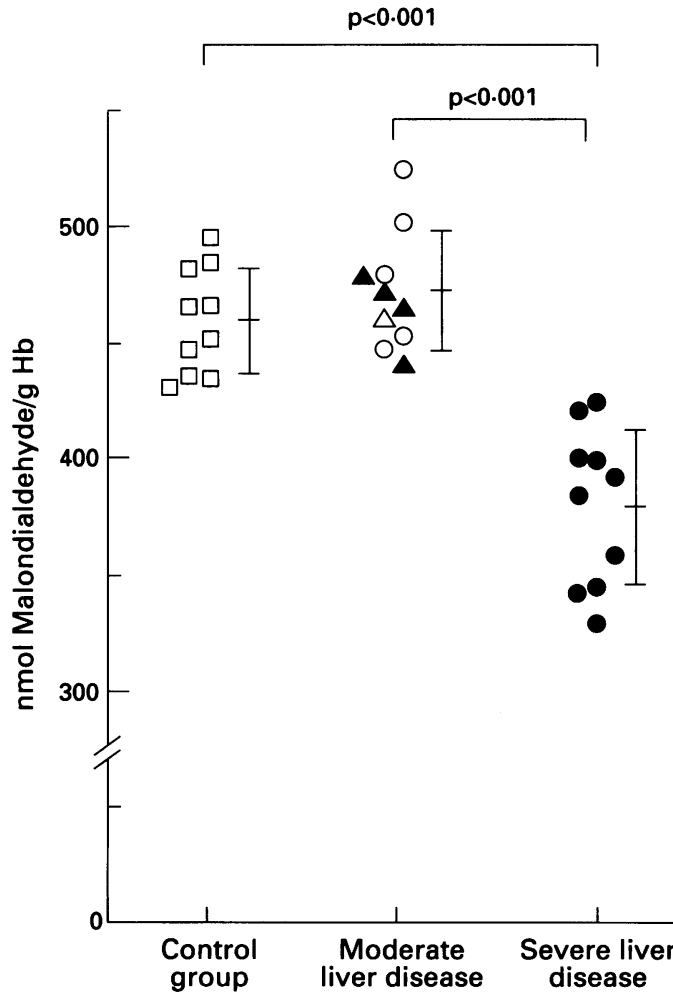

Figure 2: Lipid peroxidation in $t-B H P$ stressed erythrocytes incubated for 180 minutes related to severity of alcoholic liver disease. Control group: alcoholic patients with moderate liver disease; hepatitis ( $\Delta$ ); fatty liver ( $)$; class $A$ or $B$ cirrhosis (O): alcoholic patients with severe liver disease (O). Bars are means (SD).
$1400 \mathrm{~g}$ for 15 minutes at $4^{\circ} \mathrm{C}$ and discarding the supernatant. A 1 in 10 ( $\mathrm{vol} / \mathrm{vol})$ dilution of the packed erythrocytes was prepared in PBS and $0.1 \mathrm{ml}$ aliquots added to $5 \mathrm{ml}$ Drabkin's reagent, incubated at room temperature for 15 minutes, and the absorbance read at $540 \mathrm{~nm}$ against appropriate blanks and standards (Sigma, Poole, Dorset) to give the haemoglobin concentration. ${ }^{2122}$ To avoid changes in cell density in alcoholic patients affecting the number of erythrocytes and hence peroxidisable substrate in each incubation, incubations were standardised to contain the same amount of haemoglobin. A dilution of the packed erythrocytes was then prepared (approximately 1 in $40 \mathrm{vol} / \mathrm{vol}$ ) in PBS to give $7.5 \mathrm{mg}$ haemoglobin $/ \mathrm{ml}$.

OXYGEN RADICAL STRESSED ERYTHROCYTES

The resistance to a chemically induced free radical stress was measured inversely as the degree of lipid peroxidation. Lipid peroxidation of erythrocyte membranes was induced according to Stocks et $a l,{ }^{7}$ modified by replacing hydrogen peroxide with the more stable lipoperoxide analogue t-butyl hydroperoxide ( $t-B H P$ ), as described previously. ${ }^{2122}$ Briefly, $6 \mathrm{ml}$ aliquots of erythrocyte suspensions were stressed by adding $6 \mathrm{ml}$ $0.75 \mathrm{mmol} \mathrm{1}^{-1} \mathrm{t}$-BHP (Sigma Chemical, Poole, Dorset) at final concentrations of 3.75 $\mathrm{mg} / \mathrm{ml}$ haemoglobin and $3.75 \times 10^{-4} \mathrm{M}$ t-BHP, respectively. The erythrocytes were then incubated at $37^{\circ} \mathrm{C}$ in a shaking water bath and timed samples taken over three hours.

\section{MEASUREMENT OF LIPID PEROXIDES}

Lipid peroxidation from erythrocytes from alcoholic patients and controls was measured as malondialdehyde production as used in previous studies on erythrocytes from alcoholic patients, ${ }^{11-13}$ assessed as thiobarbituric acid reactive material, described previously. ${ }^{2122}$ Either $2 \mathrm{ml}$ of stressed erythrocyte suspension or $1 \mathrm{ml}$ of a standard solution of tetraethoxypropane (Sigma Chemical, Poole, Dorset) was added to an equal volume of 0.61 mol $1^{-1}$ trichloroacetic acid, mixed, and centrifuged at $1400 \mathrm{~g}$ for 10 minutes. Two $\mathrm{ml}$ supernatant was added to $1 \mathrm{ml} 52 \mathrm{mmol}^{-1}$ thiobarbituric acid solution in $0.1 \mathrm{~mol}^{-1}$ hydrochloric acid. Tubes were capped, mixed, and heated for 20 minutes at $95^{\circ} \mathrm{C}$, cooled, and the absorbance of the product of the malondialdehyde-thiobarbituric acid reaction at $532 \mathrm{~nm}$ recorded. The concentration of malondialdehyde in the assays was calculated using tetraethoxypropane derived malondialdehyde.

All reagents used were from $\mathrm{BDH}$, Dagenham, Essex, unless otherwise stated.

\section{STATISTICAL ANALYSIS}

Analysis of data was performed using Student's $t$ test for unpaired data, expressed as mean (SD) where appropriate. 
Results

After 60 minutes of incubation erythrocytes from patients with severe liver disease had a significantly $(p<0.02)$ increased resistance to lipid peroxidation measured as lower malondialdehyde production, compared with those from healthy subjects (Fig 1). The magnitude and statistical significance of this difference increased thereafter $(\mathrm{p}<0.001)$ (Fig 1).

The increase of lipid peroxidation with time was similar in incubations of erythrocytes from both controls and patients with moderate liver disease even after incubating for 180 minutes (Fig 2). The resistance to lipid peroxidation of erythrocytes from patients with severe liver disease was thus also significantly increased when compared with those patients with moderate liver disease after 60 minutes' incubation (216 (31) $v 243$ (34) $\mathrm{ml} / \mathrm{gHb}$; severe $v$ moderate; $\mathrm{p}<0.02)$, after 90 minutes (299 (17) v 367 (30); $\mathrm{p}<0.001), 120$ minutes (351 (37) v 444 (22); $\mathrm{p}<0.001$ ), and at 180 minutes $(\mathrm{p}<0.001)$ (Fig 2$)$.

\section{Discussion}

The resistance of erythrocytes to chemically induced lipid peroxidation was significantly increased in alcoholic patients with severe liver disease compared with those from healthy controls or patients with moderate liver disease. Apart from this difference, the production of lipid peroxides in response to t-BHP stress with time was similar in incubations of both control and alcoholic erythrocytes and comparable with that in previous studies. $^{2122}$ This increased resistance in peroxidation is in agreement with previous studies performed on alcoholic patients with cirrhosis in which lipid peroxidation was assessed as pentane production or haemolysis. ${ }^{1619}$ Moreover, although malondialdehyde production may be a poor absolute measurement of lipid peroxidation, as a relative measurement it compares well with other markers. ${ }^{23-26}$ Thus this increased resistance is not a result of the form of measurement of lipid peroxidation used.

As in other studies, ${ }^{19}$ the erythrocytes from patients with mild liver disease had a similar resistance to those from controls, suggesting that the observed difference is a result of severe liver damage rather than alcohol abuse alone. It is thus unlikely that the increased resistance arises from some protective change in vivo, and more probable that it is caused by changes in the composition of the erythrocyte rather than changes in antioxidant concentrations. Lipid peroxidation, measured as malondialdehyde, correlates well with loss of the substrate fatty acids arachidonic acid and docosahexaenoic acid, ${ }^{26}$ concentrations of which are lower in erythrocytes from alcoholic patients with cirrhosis. ${ }^{19} 27$ Thus this change in membrane fatty acids could produce the increased resistance. In conjunction with the decrease in these fatty acids, the cholesterol concentrations in erythrocytes are increased. ${ }^{1927}$ It is this increase in cholesterol, rather than the changes in fatty acids, that produces a decrease in the membrane fluidity of erythrocytes from patients with alcoholic liver disease. ${ }^{28}$

Alcohol consumption in the absence of liver disease, however, does not change erythrocyte concentrations of arachidonic acid or docosahexaenoic $\mathrm{acid}^{29-31}$ or cholesterol. ${ }^{29} 3032$ Moreover, lower concentrations of arachidonic acid and an increased resistance to lipid peroxidation are found in rats with liver disease unrelated to alcohol. ${ }^{31}$ Similarly, in humans, increased erythrocyte cholesterol occurs in a wide variety of human liver diseases. ${ }^{28}$ Thus the increased resistance to lipid peroxidation in alcoholic patients may result more from changes in erythrocyte membrane composition secondary to liver malfunction than from alcohol abuse alone.

Consumption of alcohol in the absence of severe liver disease can lower erythrocyte antioxidants ${ }^{415}$ and this could have caused the reduced resistance of erythrocytes to lipid peroxidation in studies performed on alcoholic patients with no ${ }^{13}$ or only mild ${ }^{11}$ liver disease. In both of these studies the patients were starting attendance at clinics for detoxification and so it is possible that recent, heavy alcohol consumption is required before the reduced resistance becomes apparent. Only in extreme situations in alcoholic patients, such as in the rare Zieve's syndrome, may the reduction in antioxidants be sufficient to overcome the high cholesterol and low polyunsaturated fatty acid concentrations caused by the liver disease and thus reduce resistance to lipid peroxidation. ${ }^{14}$

Thus the resistance of erythrocyte to lipid peroxidation measured in vitro may be affected by two separate events, namely changes in the cell membrane composition in response to liver disease and changes in the antioxidants in the erythrocyte in response to recent, chronic heavy intake of alcohol. These changes affect the interpretation of results of measurement of lipid peroxidation in erythrocytes.

We are grateful to the Special Trustees of St Thomas' Hospital for their continuing support. Part of this work was presented at the meeting of the Medical Research Society, January 1990, and published in abstract form (Clin Sci 1990; 78: 13).

1 Lieber CS. The biochemical basis of injury to the liver and other tissues. $N$ Engl F Med 1988; 319: 1639-50.

2 Thompson RPH. Alcohol, nutrition and the liver. Eur $\mathcal{f}$ Gastroenterol Hepatol 1990; 2: 417-21.

3 Jeffrey GP, Muller DPR, Burroughs AK, Matthews S, Kemp C, Epstein O, et al. Vitamin E deficiency and its clinical significance in adults with primary biliary cirrhosis and other forms of chronic liver disease. F Hepatol 1987; 4: 307-17.

4 Girre C, Hispard E, Therond P, Guedi S, Rourdon R, Dally $S$. Effect of abstinence from alcohol on the depression of E levels in chronic alcoholic patients. Alcohol Clin Exp Res 1990; 14: 909-13.

5 Beatti AD, Sherlock S. Ascorbic acid deficiency in liver disease. Gut 1979; 17: 571-5.

6 McClain CJ, Antonow DR, Cohen DA, Shedlofsky SI. Zinc metabolism in alcoholic liver disease. Alcoholism 1986; 10: 582-9.

7 Stocks J, Offerman EL, Modell CB, Dormandy TL. The susceptibility to autoxidation of human red cell lipids in health and disease. Br $\mathcal{F}$ Haematol 1972; 23: 713-24.

8 Dormandy Tl. The autoxidation of red cells. Br $\mathcal{f ~ H a e m a t o l}$ 1971; 20: 457-461.

9 Gordon HH, Nitowsky HM, Cornblath M. Studies of tocopherol deficiency in infants and children. Am $\mathfrak{f}$ Dis Child 1955; 90: 669-81.

10 Shah RS, Rajalakshmi R, Bhatt RV, Hazra MN, Patel BC, Swamy NB. Vitamin E status of the newborn in relation to gestational age, birth weight and maternal vitamin $\mathrm{E}$ gestational age, birth weight and
status. B F Nutr 1987; 58: 191-8.

11 Bidder TG, Jaeger PD. Malondialdehyde production by erythrocytes from alcoholics and non-alcoholic subjects. Life Sci 1982; 30: 1021-7. 
12 Uysal M, Aykac G, Kocak-Toker W, Sivas A, Yalcin S, Oz $\mathrm{H}$. Lipid peroxidation in liver, plasma and erythrocytes of rats chronically treated with alcohol. Biochem Med 1985; 34: $370-2$.

13 Uysal M, Bulur H, Erdine-Demirelli S, Demiroglu C. Erythrocyte and plasma lipid peroxides in chronic 4 Goebal KM, Goebal FD, Schubotz R, Schneider J. Red cell metabolic and membrane features in haemolytic anaemia of alcoholic liver disease

15 Capel ID, Willenbrock SJF, Jenner M, Williams DC. The effect of ethanol ingestion on the erythrocyte antioxidant defence systems of rats. Biochem Pharmacol 1980; 29: 1737-9.

16 Feher J, Lang I, Nekam K, Muzes GY, Deak GY. Effects of free radical scavengers on superoxide dismutase (SOD) enzyme in patients with alcoholic cirrhosis. Acta Med Hung 1988; 45: 265-76.

17 Wisloff $\mathrm{F}$, Boman D. Haemolytic anaemia in alcohol abuse. Acta Med Scand 1979; 205: 237-42.

18 Wood G, Lahiri S, Gorka C, Armbrecht J, Strong R. In vitro effects of ethanol on erythrocyte membrane fluidity of alcoholic patients: an electron spin resonance study. Alcoholism 1987; 11: 332-5.

19 Clemens MR, Einsele H, Remmer H, Waller HD. Decreased susceptibility of red blood cells to lipid peroxidation in patients with alcoholic liver cirrhosis. Clin Chim Acta 1988; 145: 283-8.

20 Pugh RNH, Murray-Lyon IM, Dawson JL, Pietroni MC, Williams R. Transection of the oesophagus for bleeding oesophageal varices. Br $\mathcal{F}$ Surg 1973; 60: 646-9.

21 Greenfield SM, Punchard NA, Thompson RPH. Inhibition of red cell membrane lipid peroxidation by sulphasalazine and 5-aminosalicylic acid. Gut 1991; 32: 1156-9.

22 Acharya J, Punchard NA, Taylor JA, Thompson RPH, Pearson TC. Red cell lipid peroxidation and antioxidant enzymes in iron deficiency. Eur $f$ Hepatol 1991; 47: 287-91.

23 Yasuda H, Miki M, Takenaka Y, Tamai H, Mino $M$.
Chemiluminescence from vitamin-E deficient erythrocyte membranes induced by xanthidine oxidase reaction. In: Simic MG, Taylor KA, Ward JF, von Sonntag C, eds. Oxygen radicals in biology and medicine. New York: Plenum, 1988.

24 Trotta RJ, Sullivan SG, Stern A. Lipid peroxidation and haemoglobin degradation in red blood cells exposed to haemoglobin degradation in red blood cells exposed

25 Pompella A, Maellaro E, Casini AF, Ferrali M, Ciccoli L, Comporti $M$. Measurement of lipid peroxidation in vivo: a comparison of different procedures. Lipids 1987; 22: 206-11.

26 Jordan RA, Schemkman JB. Relationship between malondialdehyde production and arachidonate consumption during NADPH supported microsomal lipid peroxidation. Biochem Pharmacol 1982; 31: 1393-400.

27 Clemens MR, Einsile H, Ladner C, Waller HD. Some new aspects on free radical reactions in red cell pathology. In: Rice-Evans C, Dormandy T, eds. Free radicals; chemistry, pathology and medicine. London: Richlieu, 1988: 383-404,

28 Owen JS, Bruckdorfer KR, Day RC, McIntyre N. Decreased erythrocyte membrane fluidity and altered lipid composition in human liver disease. $\mathcal{F}$ Lipid Res 1982; 23: $124-32$.

29 Cunnane SC, McAdoo KR, Horrobin DF. Long-term ethanol consumption in the hamster: effects on tissue lipids, fatty acids and erythrocyte hemolysis. Ann Nutr Metab 1987; 31: 265-71

30 La Droitte P, Lamboeuf Y, de Saint Blanquat G. Lipid composition of the synaptosome and erythrocyte membranes during chronic ethanol-treatment and withdrawal in the rat. Biochem Pharmacol 1984; 33: 615-24.

31 Clemens MR, Einsele H, Remmer H. Alcohol consumption and hepatic fibrosis affect the fatty acid composition of red blood cells and their susceptibility to lipid peroxidation. Arch Toxicol 1987; 60: 167-9.

32 Gastaldi $M$, Lerigue B, Feugere T, Le petit-Thevenin J, Nobile O, Boyer J. Altered acylation of erythrocyte phospholipids in alcoholism. Alcoholism 1988; 12: 356-9.

\section{.}

๑ั 